Research Article

\title{
Fractional Entropy-Based Test of Uniformity with Power Comparisons
}

\author{
Mohamed S. Mohamed $\left(\mathbb{D},{ }^{1}\right.$ Haroon M. Barakat $\mathbb{D}^{2},{ }^{2}$ Salem A. Alyami, ${ }^{3}$ \\ and Mohamed A. Abd Elgawad (iD ${ }^{4,5}$ \\ ${ }^{1}$ Department of Mathematics, Faculty of Education, Ain Shams University, Cairo 11341, Egypt \\ ${ }^{2}$ Department of Mathematics, Faculty of Science, Zagazig University, Zagazig 44519, Egypt \\ ${ }^{3}$ Department of Mathematics and Statistics, Faculty of Science, Imam Mohammad Ibn Saud Islamic University (IMSIU), \\ Riyadh 13318, Saudi Arabia \\ ${ }^{4}$ Department of Mathematics, Faculty of Science, Benha University, Benha 13518, Egypt \\ ${ }^{5}$ School of Computer Science and Technology, Wuhan University of Technology, Wuhan 430070, China
}

Correspondence should be addressed to Mohamed S. Mohamed; mohamed.said@edu.asu.edu.eg

Received 25 April 2021; Accepted 3 July 2021; Published 22 July 2021

Academic Editor: Ahmet Ocak Akdemir

Copyright (c) 2021 Mohamed S. Mohamed et al. This is an open access article distributed under the Creative Commons Attribution License, which permits unrestricted use, distribution, and reproduction in any medium, provided the original work is properly cited.

In the present paper, we use the fractional and weighted cumulative residual entropy measures to test the uniformity. The limit distribution and an approximation of the distribution of the test statistic based on the fractional cumulative residual entropy are derived. Moreover, for this test statistic, percentage points and power against seven alternatives are reported. Finally, a simulation study is carried out to compare the power of the proposed tests and other tests of uniformity.

\section{Introduction}

Rao et al. [1] suggested a nonnegative measure of uncertainty and called it the cumulative residual entropy (CRE). For any nonnegative continuous random variable (RV) $X$ with a cumulative distribution function (CDF) $F(x)=P(X<x)$, the CRE is defined by

$$
\operatorname{CRE}(F)=-\int_{0}^{\infty} \bar{F}(x) \ln (\bar{F}(x)) \mathrm{d} x
$$

where $\bar{F}(x)=1-F(x)$ is the reliability function. Rao et al. [1] revealed many salient features of the CRE. For example, the CRE possesses more general mathematical properties than the Shannon entropy, and it can be easily computed from sample data, and these computations asymptotically converge to the true values. Moreover, the CRE deals with the quantity of information in residual life. For the standard uniform distribution, denoted by $U(0,1)$, Rao et al. [1] showed that the value of the CRE is $1 / 4$. The literature abounds with many different results for Shannon's entropy and its modifications. Interested readers may refer to [1-17].

Xiong et al. [16] suggested the fractional cumulative residual entropy (FCRE) to extend the CRE to the case of fractional order. For any $0 \leq q \leq 1$, the FCRE for the RV $X$ is defined by

$$
\mathrm{CRE}^{q}(F)=\int_{0}^{\infty} \bar{F}(x)[-\ln (\bar{F}(x))]^{q} \mathrm{~d} x .
$$

The measure $\operatorname{CRE}^{q}(F)$ is a nonadditive and nonnegative. Moreover, it is a convex function of the parameter $q$, $\mathrm{CRE}^{0}(F)=\mathbb{E}(X)$, and $\mathrm{CRE}^{1}(F)=\operatorname{CRE}(F)$. Xiong et al. [16] derived the FCRE for some well-known distributions; for example, FCRE of the $\operatorname{CDF} U(0,1)$ is $\Gamma(q+1) / 2^{q+1}$.

Misagh et al. [15] proposed a weighted form of CRE, which is shift-dependent. This information-theoretic uncertainty measure is called the weighted cumulative residual entropy (WCRE), and it is defined by 


$$
\mathrm{CRE}_{w}(F)=-\int_{0}^{\infty} x \bar{F}(x) \ln (\bar{F}(x)) \mathrm{d} x
$$

Later, Mirali et al. [12] and Mirali and Baratpour [13] studied several properties of this measure including its dynamic version. It is easy to observe that the WCRE of the $U(0,1)$ is $5 / 36$.

Stephens [18] offered a practical guide to goodness-of-fit tests using statistics based on the empirical CDF. Moreover, in [18], the power comparisons of some uniformity tests were carried out. Dudewicz and Van der Meulen [9] investigated the power properties of an entropy-based test when used for testing uniformity. Moreover, via a comparison with other tests of uniformity, Dudewicz and Van der Meulen [9] showed that the entropy-based test possesses good power properties for many alternatives. Noughabi [14] constructed a test for uniformity based on the CRE and studied some of its properties. Moreover, he reported the percentage points and power comparison against seven alternative distributions. As a natural extension of the results obtained by Noughabi [14], we study the FCRE and WCRE for testing the uniformity. A result of a simulation study shows that the test based on FCRE and WCRE is competitive with the test based on CRE in terms of power. This fact gives a satisfactory motivation of our study.

Throughout this paper, we obtain the percentage points under the WCRE and FCRE by using the Monte Carlo method via the simulation and the normality asymptotic, as well as the beta approximation, respectively. Moreover, a power comparison is performed between the FCRE and WCRE and other tests. The rest of this work is systematic as follows. In Section 2, we introduce the FCRE test statistic for uniformity and discuss some of its properties. In Section 3, we propose the methods of finding the percentage points of FCRE and illustrate the WCRE test statistics for uniformity. In addition, we calculate the percentage points of FCRE and WCRE. Then, in Section 4, we use Monte Carlo simulation to perform the power comparison of uniformity of different tests against seven alternative distributions. Section 5 is devoted to the conclusions. Everywhere in what follows, the symbols $(\underset{n}{\stackrel{p}{\longrightarrow}}),(\underset{n}{\stackrel{d}{\longrightarrow}})$ and $(\stackrel{\text { a.s. }}{\longrightarrow})$ stand for convergence in probability, convergence in distribution, and almost surely, as $n \longrightarrow \infty$.

\section{Theoretical Aspects and Test Statistic}

To establish our test of the null hypothesis $H_{0}$, we need the following theorem, which shows that, for a CDF with support $[0,1]$, one always has $0 \leq \operatorname{CRE}^{q}(F) \leq e^{-q}$, and for the distribution $U(0,1)$, we have FCRE $=\Gamma(q+1) / 2^{q+1}$, and this value is uniquely attained by the uniform distribution, whenever $q$ is fixed.

Theorem 1. Let $X$ be a nonnegative $R V$ with an absolutely continuous CDF $F$ with a support $[0,1]$. From (2), it holds $0 \leq C R E^{q}(F) \leq e^{-q}$, and $\operatorname{CRE}^{q}(F)=\Gamma(q+1) / 2^{q+1}$ is uniquely acquired by the distribution $U(0,1)$.
Proof. Since $0 \leq \bar{F}(x)[-\ln (\bar{F}(x))]^{q} \leq 1$, and the function $f(x)=x(-\ln x)^{q}$ has a maximum at $x=e^{-q}, 0<x \leq 1$, we get $0 \leq \operatorname{CRE}^{q}(F) \leq e^{-q}$. On the other hand, using the strict convexity of $f(x)=x(-\ln x)^{q}$, it is easy to see that FCRE is a concave function of distribution (with support $[0,1]$ ). This shows that $\operatorname{CRE}^{q}(F)=\Gamma(q+1) / 2^{q+1}$ is uniquely acquired by the distribution $U(0,1)$. This completes the proof.

Let $X_{1}, X_{2}, \ldots, X_{n}$ be a random sample with a continuous $\mathrm{CDF} F$, with support $[0,1]$. Furthermore, let $X_{(1)} \leq X_{(2)} \leq \cdots \leq X_{(n)}$ be the corresponding order statistics $X_{1}, X_{2}, \ldots, X_{n}$. According to (2), we can obtain the empirical FCRE as an estimator of $\operatorname{FCRE}(F)$ by

$$
\operatorname{CRE}^{q}\left(F_{n}\right)=\int_{0}^{\infty} \bar{F}_{n}(x)\left[-\ln \left(\bar{F}_{n}(x)\right)\right]^{q} \mathrm{~d} x,
$$

where $\bar{F}_{n}(x)=1-F_{n}(x)$ and $F_{n}(x)$ is the empirical CDF, which is defined by

$$
F_{n}(x)=\sum_{i=1}^{n-1} \frac{i}{n} I_{\left[X_{(i)}, X_{(i+1)}\right)}(x)+I_{\left[X_{(n)}, \infty\right)}(x), \quad x \in \Re,
$$

where $I_{A}(x)$ is the indicator function, i.e., $I_{A}(x)=1, x \in A$; $I_{A}(x)=0, x \notin A$.

To perform a consistent test of the hypothesis of uniformity, we suggest the consistent statistic test

$$
\begin{aligned}
R_{n}^{q}=\operatorname{CRE}^{q}\left(F_{n}\right) & =\sum_{i=1}^{n-1}\left(1-\frac{i}{n}\right)\left(-\ln \left(1-\frac{i}{n}\right)\right)^{q}\left(X_{(i+1)}-X_{(i)}\right) \\
& =\sum_{i=1}^{n-1} A_{i} W_{i},
\end{aligned}
$$

where $A_{i}=(1-(i / n))(-\ln (1-(i / n)))^{q}$ and $W_{i}=\left(X_{(i+1)}-\right.$ $\left.X_{(i)}\right), i=1,2, \ldots, n-1,0 \leq q \leq 1$.

Xiong et al. [16] proved that $\operatorname{CRE}^{q}\left(F_{n}\right) \stackrel{p}{\rightarrow} \operatorname{CRE}^{q}(F)$. Moreover, under the null hypothesis $H_{0}$, we get $R_{n}^{q} \underset{n}{\stackrel{p}{\longrightarrow}} \Gamma$ $(q+1) / 2^{q+1}$. On the other hand, under the alternative hypothesis (that $F$ is any continuous CDF with support [0, 1], which is not the uniform), we have $\operatorname{CRE}^{q}\left(F_{n}\right) \underset{n}{\stackrel{p}{\longrightarrow}} r$, where $r$ is a smaller or larger number than $\Gamma(q+1) / 2^{q+1}$.

Theorem 2. The test based on the sample estimate $R_{n}^{q}$ is consistent.

Proof. From Glivenko-Cantelli theorem (see Tucker [19]), we have $\sup _{t}\left|F_{n}(t)-F(t)\right| \underset{n}{\stackrel{\text { a.s. }}{\longrightarrow}} 0$. On the other hand, Theorem 3 in Xiong et al. [16] asserts that $\operatorname{CRE}^{q}\left(F_{n}\right) \underset{n}{\stackrel{\text { a.s. }}{\longrightarrow}} \operatorname{CRE}^{q}(F)$, which proves the theorem.

Theorem 3. Suppose that the random sample $X_{1}, X_{2}, \ldots, X_{n}$ has been drawn from an unknown continuous CDF $F$ defined on $[0,1]$. Then, from (6), we have $0 \leq R_{n}^{q} \leq e^{-q}$.

Proof. Since the function $f(p)=p(-\ln p)^{q}, 0<p<1$, has a maximum value at $e^{-q}, 0 \leq q \leq 1$; therefore, 


$$
\begin{aligned}
0 \leq R_{n}^{q}=\operatorname{CRE}^{q}\left(F_{n}\right) & =\sum_{i=1}^{n-1}\left(1-\frac{i}{n}\right)\left(-\ln \left(1-\frac{i}{n}\right)\right)^{q}\left(X_{(i+1)}-X_{(i)}\right) \\
& \leq \sum_{i=1}^{n-1} e^{-q}\left(X_{(i+1)}-X_{(i)}\right)=e^{-q}\left(X_{(n)}-X_{(1)}\right) \leq e^{-q}
\end{aligned}
$$

This completes the proof of the theorem.

Theorem 4. Under $H_{0}$, from (6), the mean and the variance of $R_{n}^{q}$ are, respectively,

$$
\begin{aligned}
\mathbb{E}\left(R_{n}^{q}\right) & =\frac{1}{n+1} \sum_{i=1}^{n-1} A_{i}, \\
\operatorname{Var}\left(R_{n}^{q}\right) & =\frac{n}{(n+1)^{2}(n+2)} \sum_{i=1}^{n-1} A_{i}^{2} .
\end{aligned}
$$

Proof. The proof directly follows by noting that, for any $i=1,2, \ldots, n-1$, the RV $W_{i}=\left(X_{(i+1)}-X_{(i)}\right)$, based on the $\operatorname{CDF} U(0,1)$, has beta distribution, i.e., $W_{i} \sim \operatorname{Beta}(1, n)$ (cf. [20]). This completes the proof.

Remark 1. Under $H_{0}$, from (6), (8), and (9), we have $\lim _{n \longrightarrow \infty} \mathbb{E}\left(R_{n}^{q}\right)=\Gamma(q+1) / 2^{q+1}=\operatorname{CRE}^{q}(U)$ and $\lim _{n \rightarrow \infty}$ $\operatorname{Var}\left(R_{n}^{q}\right)=0$, where $\operatorname{CRE}^{q}(U)$ is the FCRE of the CDF $U(0,1)$.

The critical region, which describes the test procedure, is given by the following two inequalities:

$$
\begin{aligned}
\operatorname{CRE}^{q}\left(F_{n}\right) & \leq \operatorname{CRE}_{\alpha / 2}^{* q}:=\text { lower or } \operatorname{CRE}^{q}\left(F_{n}\right) \\
& \geq \operatorname{CRE}_{1-(\alpha / 2)}^{* q}:=\text { upper, }
\end{aligned}
$$

where $\alpha$ is the desired level of significance, and $\mathrm{CRE}_{\alpha}^{* q}$ is the $\alpha$-quantile of the asymptotic, or approximated, CDF of the test statistic $\mathrm{CRE}^{q}\left(F_{n}\right)$, under $H_{0}$. In the next section, we derive the asymptotic and approximated CDF of the test statistic $\operatorname{CRE}^{q}\left(F_{n}\right)$. These quantiles are computed by using the Monte Carlo method.

\section{Percentage Points of the Test Statistic}

In this section, we obtain the asymptotic distribution of $R_{n}^{q}$ under $H_{0}$. From (6), we can write $R_{n}^{q}=\sum_{i=1}^{n-1} T_{i}$, where $T_{i}=A_{i} W_{i}, i=1,2, \ldots, n-1$, and $W_{i} \sim \operatorname{Beta}(1, n)$. Thus, we can see that $T_{i}$ 's have the following probability density function (PDF):

$$
f_{T_{i}}(t)=\frac{n}{A_{i}}\left(1-\frac{t}{A_{i}}\right)^{n-1}, \quad i=1,2, \ldots, n-1 .
$$

The mean and variance of $T_{i}$ are, respectively,

$$
\begin{aligned}
\mu_{i} & =\mathbb{E}\left(T_{i}\right)=A_{i} \mathbb{E}\left(W_{i}\right)=\frac{A_{i}}{n+1}, \\
\sigma_{i}^{2} & =\operatorname{Var}\left(T_{i}\right)=A_{i}^{2} \operatorname{Var}\left(W_{i}\right)=\frac{n A_{i}^{2}}{(n+1)^{2}(n+2)} .
\end{aligned}
$$

According to Lyapunov central limit theorem (see Billingsley [21]), we have $\sum_{i=1}^{n-1}\left(T_{i}-\mu_{i}\right) / \sqrt{\sum_{i=1}^{n-1} \sigma_{i}^{2}}=\left(R_{n}^{q}-\right.$ $\left.\mathbb{E}\left(R_{n}^{q}\right) / \sqrt{\operatorname{Var}\left(R_{n}^{q}\right)}\right) \underset{n}{\stackrel{d}{\longrightarrow}} \mathcal{N}$, where $\mathcal{N}$ is the standard normal RV (in the sequel, the standard normal distribution will be denoted by $N(0,1))$. Therefore, under $H_{0}$, the percentage point ( $\alpha$-quantile) $\mathrm{CRE}_{\alpha}^{* q}$ is approximated according to the asymptotic normality of $R_{n}^{q}$ for large $n$ by

$$
\mathrm{CRE}_{\alpha}^{* q}=\mathbb{E}\left(R_{n}^{q}\right)+\sqrt{\operatorname{Var}\left(R_{n}^{q}\right)} Z_{\alpha},
$$

where $Z_{\alpha}$ corresponds to the quantile $(\alpha \times 100)$ of the CDF $N(0,1)$.

Johannesson and Giri [22] proposed an approximation of the CDF of linear combination of the finite number of beta RVs. Noughabi [14] used this approximation to obtain approximately the percentage points of the CRE for finite $n$. By adopting the same procedure of Noughabi [14], we can obtain an approximation of $R_{n}^{q}$ for finite $n$ as follows:

$$
R_{n}^{q} \approx\left(\sum_{i=1}^{n-1} A_{i}\right) Y
$$

where the RV $Y$ has $\operatorname{Beta}(a, b)$ distribution,

$$
\begin{aligned}
& a=\frac{(n+2)\left(\sum_{i=1}^{n-1} A_{i}\right)^{2}}{(n+1)\left(\sum_{i=1}^{n-1} A_{i}^{2}\right)}-\frac{1}{n+1}, \\
& b=\frac{n}{n+1}\left(\frac{(n+2)\left(\sum_{i=1}^{n-1} A_{i}\right)^{2}}{\sum_{i=1}^{n-1} A_{i}^{2}}-1\right),
\end{aligned}
$$

and $A_{i}=(1-(i / n))(-\ln (1-(i / n)))^{q}, 0 \leq q \leq 1, i=1,2, \ldots$, $n-1$. According to (14), the mean and variance of $R_{n}^{q}$ are, respectively,

$$
\begin{aligned}
\mathbb{E}\left(R_{n}^{q}\right) & =\left(\sum_{i=1}^{n-1} A_{i}\right) \frac{a}{a+b} \\
\operatorname{Var}\left(R_{n}^{q}\right) & =\left(\sum_{i=1}^{n-1} A_{i}\right)^{2} \frac{a b}{(a+b)^{2}(a+b+1)} .
\end{aligned}
$$

Now, by using this approximation of $R_{n}^{q}$, the quantiles of order $\alpha / 2$ and $1-(\alpha / 2)$ of the approximated CDF of the test statistic $\mathrm{CRE}^{q}\left(F_{n}\right)$ under $H_{0}$ are, respectively,

$$
\begin{aligned}
& \text { lower }:=\left(\sum_{i=1}^{n-1} A_{i}\right) F^{-1}\left(\frac{\alpha}{2}\right), \\
& \text { upper }:=\left(\sum_{i=1}^{n-1} A_{i}\right) F^{-1}\left(1-\frac{\alpha}{2}\right),
\end{aligned}
$$


where $F^{-1}($.$) is the quantile function of the \operatorname{CDF} F, F$ is the $\operatorname{Beta}(a, b)$ distribution, and $a$ and $b$ are defined in (15).

3.1. Empirical Weighted Cumulative Residual Entropy. From (3), Misagh et al. [15] proposed the empirical WCRE by

$$
\begin{aligned}
\operatorname{CRE}_{w}\left(F_{n}\right) & =-\sum_{i=1}^{n-1}\left(\frac{X_{(i+1)}^{2}-X_{(i)}^{2}}{2}\right)\left(1-\frac{i}{n}\right) \ln \left(1-\frac{i}{n}\right) \\
& =\sum_{i=1}^{n-1} A_{i} U_{i},
\end{aligned}
$$

where $A_{i}=X_{(i+1)}^{2}-X_{(i)}^{2} / 2, U_{i}=-(1-(i / n)) \ln (1-(i / n))$, $i=1,2, \ldots, n-1$.

We suggest the following statistic of a consistent test based on (18):

$$
T_{n}^{w}=\operatorname{CRE}_{w}\left(F_{n}\right)=\sum_{i=1}^{n-1} A_{i} U_{i} .
$$

Theorem 5. The test based on the sample estimate $T_{n}^{w}$ is consistent.

Proof. From Mirali et al. [12] and by using Glivenko-Cantelli theorem, (see Tucker [19]), we have $\mathrm{CRE}_{w}\left(F_{n}\right) \underset{n}{\stackrel{\text { a.s. }}{\longrightarrow}} \operatorname{CRE}_{w}(F)$, which proves the theorem.

Theorem 6. Let $X_{1}, X_{2}, \ldots, X_{n}$ be a random sample drawn from an unknown continuous CDF F defined on $[0,1]$. Then, from (18), we get $0 \leq T_{n}^{w} \leq 1 / 2 e$.

Proof. Since the function $f(p)=-p \ln p, 0<p<1$, has a maximum value at $1 / e$; therefore,

$$
\begin{aligned}
0 \leq T_{n}^{w}=\mathrm{CRE}_{w}\left(F_{n}\right) & =-\sum_{i=1}^{n-1}\left(\frac{X_{(i+1)}^{2}-X_{(i)}^{2}}{2}\right)\left(1-\frac{i}{n}\right) \ln \left(1-\frac{i}{n}\right) \\
& \leq \frac{1}{2} \sum_{i=1}^{n-1} \frac{1}{e}\left(X_{(i+1)}^{2}-X_{(i)}^{2}\right)=\frac{1}{2 e}\left(X_{(n)}^{2}-X_{(1)}^{2}\right) \leq \frac{1}{2 e} .
\end{aligned}
$$

This completes the proof.

3.2. Percentage Points. We generate 50, 000 samples of size $n$, where $n=10,20,30,40,50,70,100$, from $U(0,1)$. Using (6), the test statistic $R_{n}^{q}$ is estimated by the empirical $R_{n}^{q}$ for each sample and the same for $T_{n}^{w}$. Moreover, we can see that $\mathrm{CRE}^{0.1}(U)=0.4438, \quad \mathrm{CRE}^{0.5}(U)=0.3133, \quad \mathrm{CRE}^{0.9}(U)=$ 0.2576 and $\operatorname{CRE}_{w}(U)=0.1388$, where $\operatorname{CRE}^{q}(U)$ and $\mathrm{CRE}_{w}(U)$ are the FCRE and WCRE of the CDF $U(0,1)$, respectively. Consequently, for $R_{n}^{q}$, we present the percentage points of the Monte Carlo method, asymptotic normality, and beta approximation by using (10), (13), and (17), respectively. The result of this study is given in Table 1, where we note that the difference between the percentage points decreases when $n$ increases. Besides, for $R_{n}^{q}$, the accuracy of the Monte Carlo method is more than the other two methods.

Figures 1-4 represent the empirical PDF's of the test statistics using Monte Carlo samples with $n=10,20,30,50,100$. When $n$ increases, it turned out that the test statistics are nearer to the exact values, which implies that the bias and the variance decrease with increasing $n$.

\section{Power Analysis}

In this section, we study the power test of Monte Carlo study under alternative distributions. The power of $R_{n}^{q}$ is estimated by the proportion of the generated samples falling into the critical region. Under seven alternative distributions, the power of the test statistic $R_{n}^{q}$ is calculated by the Monte Carlo study of generating 50,000 samples each of size $n$, where $n=20,30,50$. The alternative CDFs proposed by Stephens [18] in power study of uniformity tests are as follows:

$$
\begin{aligned}
& A_{l}: F(y)=1-(1-y)^{l}, \quad 0 \leq y \leq 1, l=1.5,2, \\
& B_{l}: F(y)= \begin{cases}2^{l-1} y^{l}, & 0 \leq y \leq 0.5, \\
1-2^{l-1}(1-y)^{l}, & 0.5 \leq y \leq 1, l=1.5,2,3,\end{cases} \\
& C_{l}: F(y)= \begin{cases}0.5-2^{l-1}(0.5-y)^{l}, & 0 \leq y \leq 0.5, \\
0.5+2^{l-1}(y-0.5)^{l}, & 0.5 \leq y \leq 1, \text { for } l=1.5,2 .\end{cases}
\end{aligned}
$$

In Table 2, based on the Monte Carlo study, we recorded the power values of the proposed test statistics $R_{n}^{q}, T_{n}^{w}$, Kolmogorov-Smirnov (K-S), Kuiper (V), Cramer-von Mises $\left(W^{2}\right)$, Watson $\left(U^{2}\right)$, and Anderson-Darling $\left(A^{2}\right)$, for $n=$ $10,20,30$ and $\alpha=0.05$. From Table 2 , we can conclude the following:

(1) If $q$ increases and tends to $1(q \longrightarrow 1)$, the power of $C R E^{q}$ test, for alternative $A_{l}\left(B_{l}\right)\left(C_{l}\right)$, decreases (increases) (increases), and vice versa, if $q$ decreases and tends to $0(q \longrightarrow 0)$.

(2) If $q \longrightarrow 1$, the $\mathrm{CRE}^{q}$ test, for alternative $A_{l}\left(B_{l}\right)$, gives the worst (best) performance compared with the other tests.

(3) To compare the performance between $\mathrm{CRE}^{q}$ and $\mathrm{CRE}_{w}$ tests, we observe that:

(a) For the alternative $A_{l}, q \longrightarrow 1, \mathrm{CRE}_{w}$ performs better than $\mathrm{CRE}^{q}$ and vice versa if $q \longrightarrow 0, n$ increases.

(b) For the alternative $B_{l}, q \longrightarrow 1, \mathrm{CRE}^{q}$ performs better than $\mathrm{CRE}_{w}$, and vice versa, if $q \longrightarrow 0, n$ increases.

(c) For the alternative $C_{l}, q \longrightarrow 0, \mathrm{CRE}_{w}$ performs better than $\mathrm{CRE}^{q}$, and vice versa, if $q \longrightarrow 1$.

Stephens [18] noted that $V$ and $U^{2}$ tests will reveal a change at variance. Therefore, we observe the following:

(1) For alternative $A_{l}, q \longrightarrow 0, \mathrm{CRE}^{q}$ performs better than $V$ and $U^{2}$, and vice versa, if $q \longrightarrow 1$. 
TABle 1: Percentage points of the proposed test statistics $R_{n}^{q}$ and $T_{n}^{w}$ at level $\alpha=0.05$.

\begin{tabular}{|c|c|c|c|c|c|c|c|c|c|}
\hline \multirow{3}{*}{$n$} & \multirow{3}{*}{$q$} & \multicolumn{6}{|c|}{$R_{n}^{q}$} & \multirow{2}{*}{\multicolumn{2}{|c|}{$T_{n}^{w}$}} \\
\hline & & \multicolumn{2}{|c|}{ Monte Carlo method } & \multicolumn{2}{|c|}{ Normal approximation } & \multicolumn{2}{|c|}{ Beta approximation } & & \\
\hline & & Upper & Lower & Upper & Lower & Upper & Lower & Upper & Lower \\
\hline \multirow{3}{*}{10} & 0.1 & 0.5131 & 0.2282 & 0.6165 & 0.1293 & 0.6495 & 0.1672 & \multirow{3}{*}{0.1574} & \multirow{3}{*}{0.0669} \\
\hline & 0.5 & 0.3522 & 0.1818 & 0.4481 & 0.1065 & 0.47003 & 0.1315 & & \\
\hline & 0.9 & 0.2964 & 0.14901 & 0.3732 & 0.0873 & 0.3916 & 0.1084 & & \\
\hline \multirow{3}{*}{20} & 0.1 & 0.50608 & 0.3041 & 0.6012 & 0.2144 & 0.6217 & 0.2368 & \multirow{3}{*}{0.1544} & \multirow{3}{*}{0.0957} \\
\hline & 0.5 & 0.3458 & 0.2341 & 0.4282 & 0.1632 & 0.4415 & 0.1777 & & \\
\hline & 0.9 & 0.2892 & 0.1901 & 0.3549 & 0.1335 & 0.3661 & 0.1458 & & \\
\hline \multirow{3}{*}{30} & 0.1 & 0.4995 & 0.3357 & 0.58407 & 0.2556 & 0.5987 & 0.2715 & \multirow{3}{*}{0.15275} & \multirow{3}{*}{0.1068} \\
\hline & 0.5 & 0.3422 & 0.2544 & 0.4135 & 0.19007 & 0.423 & 0.2002 & & \\
\hline & 0.9 & 0.2853 & 0.2066 & 0.34207 & 0.1555 & 0.35009 & 0.1641 & & \\
\hline \multirow{3}{*}{40} & 0.1 & 0.4945 & 0.3541 & 0.5709 & 0.2809 & 0.5824 & 0.2931 & \multirow{3}{*}{0.1515} & \multirow{3}{*}{0.1126} \\
\hline & 0.5 & 0.3393 & 0.2646 & 0.40309 & 0.2064 & 0.4104 & 0.2142 & & \\
\hline & 0.9 & 0.2823 & 0.2153 & 0.3331 & 0.16902 & 0.3393 & 0.1756 & & \\
\hline \multirow{3}{*}{50} & 0.1 & 0.4905 & 0.3653 & 0.5607 & 0.2983 & 0.5701 & 0.3082 & \multirow{3}{*}{0.1506} & \multirow{3}{*}{0.1163} \\
\hline & 0.5 & 0.3374 & 0.2712 & 0.3953 & 0.2177 & 0.4013 & 0.2241 & & \\
\hline & 0.9 & 0.2808 & 0.2207 & 0.3265 & 0.1783 & 0.3316 & 0.1837 & & \\
\hline \multirow{3}{*}{70} & 0.1 & 0.4854 & 0.3801 & 0.5461 & 0.3213 & 0.553 & 0.3285 & \multirow{3}{*}{0.1492} & \multirow{3}{*}{0.1206} \\
\hline & 0.5 & 0.3345 & 0.2796 & 0.3844 & 0.2326 & 0.3888 & 0.2372 & & \\
\hline & 0.9 & 0.2776 & 0.2276 & 0.3172 & 0.1906 & 0.32103 & 0.1945 & & \\
\hline \multirow{3}{*}{100} & 0.1 & 0.4799 & 0.3919 & 0.5317 & 0.3418 & 0.5367 & 0.3469 & \multirow{3}{*}{0.14805} & \multirow{3}{*}{0.1242} \\
\hline & 0.5 & 0.3317 & 0.2859 & 0.37404 & 0.2459 & 0.3772 & 0.2492 & & \\
\hline & 0.9 & 0.27508 & 0.2335 & 0.3085 & 0.2016 & 0.3112 & 0.2044 & & \\
\hline
\end{tabular}

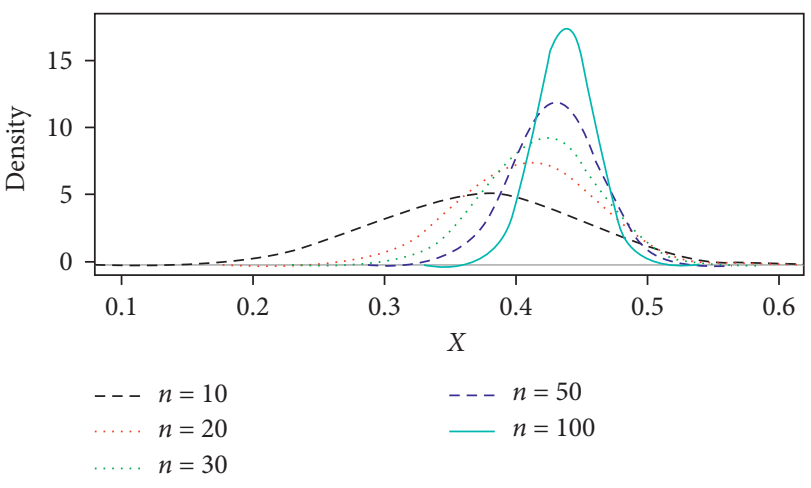

FIgURE 1: The estimated PDF's of $R_{n}^{0.1}$ based on $U(0,1)$.

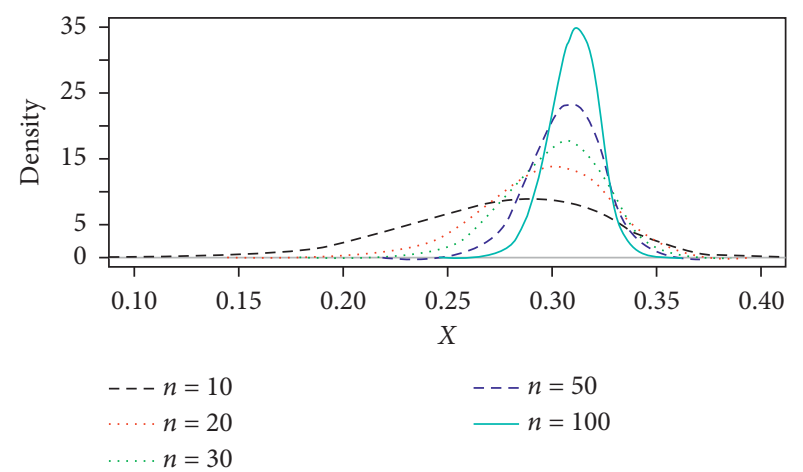

Figure 2: The estimated PDF's of $R_{n}^{0.5}$ based on $U(0,1)$.

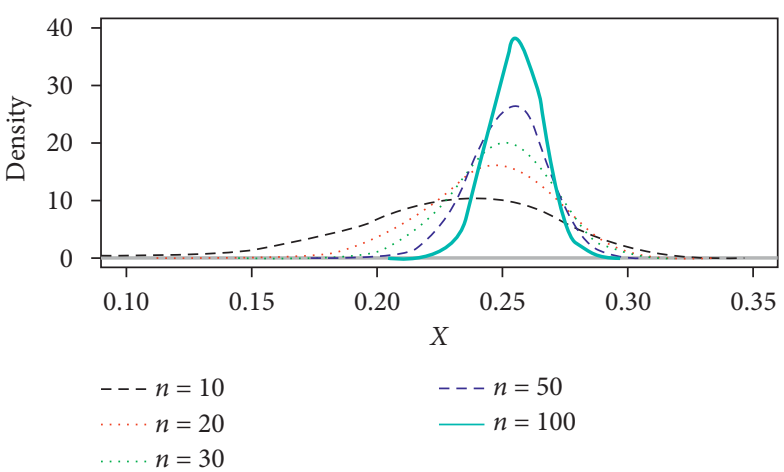

Figure 3: The estimated PDF's of $R_{n}^{0.9}$ based on $U(0,1)$.

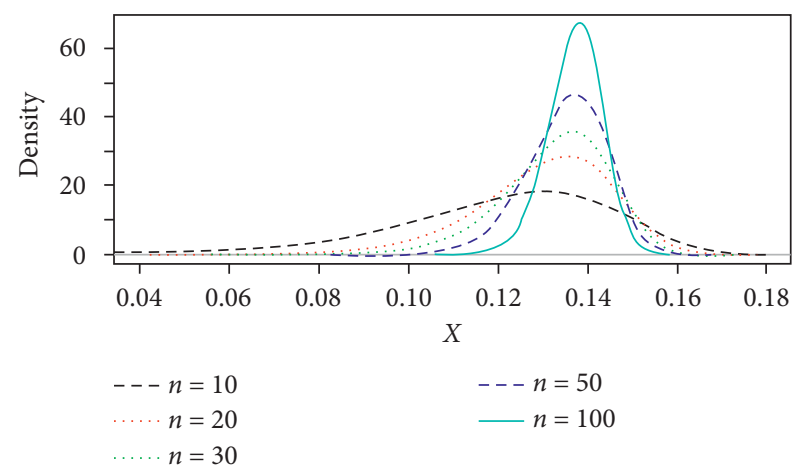

FIgURE 4: The estimated PDF's of $T_{n}^{w}$ based on $U(0,1)$. 
Table 2: Power estimates of the tests at level $\alpha=0.05$.

\begin{tabular}{|c|c|c|c|c|c|c|c|c|c|c|}
\hline$n$ & Alternative & 0.1 & $\begin{array}{l}R_{n}^{q} \\
0.5\end{array}$ & 0.9 & $T_{n}^{w}$ & K-S & $V$ & $W^{2}$ & $U^{2}$ & $A^{2}$ \\
\hline \multirow{7}{*}{10} & $A_{1.5}$ & 0.10708 & 0.0908 & 0.07208 & 0.14002 & 0.12616 & 0.0756 & 0.1456 & 0.07776 & 0.1877 \\
\hline & $A_{2}$ & 0.2771 & 0.2327 & 0.15104 & 0.3414 & 0.30298 & 0.1631 & 0.3551 & 0.16308 & 0.4761 \\
\hline & $B_{1.5}^{2}$ & 0.10406 & 0.1314 & 0.1302 & 0.0896 & 0.07352 & 0.0971 & 0.0741 & 0.1017 & 0.1349 \\
\hline & $B_{2}$ & 0.2427 & 0.3379 & 0.3357 & 0.21402 & 0.1184 & 0.2307 & 0.1104 & 0.2481 & 0.3269 \\
\hline & $B_{3}$ & 0.5763 & 0.7662 & 0.7723 & 0.5516 & 0.2424 & 0.5394 & 0.2154 & 0.5699 & 0.72308 \\
\hline & $C_{1.5}$ & 0.0843 & 0.119 & 0.1217 & 0.0942 & 0.0342 & 0.0974 & 0.0239 & 0.1031 & 0.0222 \\
\hline & $C_{2}$ & 0.1354 & 0.2478 & 0.2543 & 0.1723 & 0.0402 & 0.2333 & 0.01114 & 0.2475 & 0.00924 \\
\hline \multirow{7}{*}{20} & $A_{1.5}$ & 0.2496 & 0.1975 & 0.0909 & 0.2543 & 0.2179 & 0.1226 & 0.25208 & 0.1225 & 0.3235 \\
\hline & $A_{2}$ & 0.6679 & 0.5672 & 0.2654 & 0.637 & 0.5616 & 0.3486 & 0.6241 & 0.3358 & 0.7538 \\
\hline & $B_{1.5}$ & 0.1449 & 0.2828 & 0.2797 & 0.2047 & 0.0869 & 0.1634 & 0.0781 & 0.1786 & 0.1774 \\
\hline & $B_{2}$ & 0.38602 & 0.7223 & 0.7222 & 0.5511 & 0.1849 & 0.4647 & 0.162 & 0.5067 & 0.52802 \\
\hline & $B_{3}$ & 0.8104 & 0.9923 & 0.9931 & 0.954 & 0.4588 & 0.8711 & 0.4615 & 0.8978 & 0.93998 \\
\hline & $C_{1.5}^{3}$ & 0.0941 & 0.1979 & 0.2101 & 0.1516 & 0.0509 & 0.1621 & 0.02406 & 0.1791 & 0.0213 \\
\hline & $C_{2}$ & 0.1509 & 0.4551 & 0.4833 & 0.3296 & 0.1162 & 0.4633 & 0.0462 & 0.5048 & 0.0338 \\
\hline \multirow{7}{*}{30} & $A_{1.5}$ & 0.4045 & 0.3273 & 0.1158 & 0.3686 & 0.3144 & 0.18002 & 0.366 & 0.1721 & 0.4498 \\
\hline & $A_{2}$ & 0.8856 & 0.8061 & 0.3854 & 0.8285 & 0.7522 & 0.5447 & 0.8105 & 0.5071 & 0.8973 \\
\hline & $B_{1.5}^{2}$ & 0.1707 & 0.4466 & 0.4481 & 0.3331 & 0.1021 & 0.2477 & 0.0873 & 0.2667 & 0.2281 \\
\hline & $B_{2}$ & 0.4794 & 0.9148 & 0.9173 & 0.7985 & 0.2706 & 0.6695 & 0.25108 & 0.7076 & 0.7002 \\
\hline & $B_{3}$ & 0.9007 & 0.99994 & 0.99998 & 0.9983 & 0.6701 & 0.97506 & 0.7237 & 0.9819 & 0.99104 \\
\hline & $C_{1.5}$ & 0.1021 & 0.2759 & 0.2938 & 0.2084 & 0.07 & 0.2492 & 0.0303 & 0.2678 & 0.0271 \\
\hline & $C_{2}$ & 0.1627 & 0.6123 & 0.6513 & 0.4736 & 0.2077 & 0.6711 & 0.1258 & 0.7111 & 0.1105 \\
\hline
\end{tabular}

(2) For the alternative $B_{l}, q \longrightarrow 1, \mathrm{CRE}^{q}$ performs better than $V$ and $U^{2}$, and vice versa, if $q \longrightarrow 0, n$ increases.

(3) For the alternative $C_{l}, q \longrightarrow 0, \mathrm{~V}$ and $U^{2}$ performs better than $\mathrm{CRE}^{q}$.

(4) $\mathrm{CRE}_{w}$ performs better than $V$ and $U^{2}$ against the alternative $A_{l}$.

(5) $\mathrm{CRE}_{w}$ performs better than $V$ and $U^{2}$ against the alternative $B_{l}, n$ increases. But, $V$ and $U^{2}$ perform better than $\mathrm{CRE}_{w}$ against the alternative $C_{l}$.

Consequently, based on alternatives with a change toward a smaller variance, the tests $\mathrm{CRE}_{w}$ and $\mathrm{CRE}^{q}, q \longrightarrow 1$, are the best. Meanwhile, under alternatives with a change toward a larger variance, the tests $\mathrm{CRE}_{w}$ and $\mathrm{CRE}^{q}, q \longrightarrow 0$, are weaker.

\section{Conclusion}

For the CDFs with support $[0,1]$, we exhibited that the values of $\mathrm{CRE}^{q}$ and $\mathrm{CRE}_{w}$ are within $\left[0, e^{-q}\right]$ and $[0,1 / 2 e]$, respectively. Moreover, the test of uniformity was proposed by calculating the percentage points and power analysis of $\mathrm{CRE}^{q}$ and $\mathrm{CRE}_{w}$. Besides, for $\mathrm{CRE}^{q}$, we obtained the percentage points by using the Monte Carlo method via the simulation and the normality asymptotic, as well as the beta approximation. Moreover, for $\mathrm{CRE}_{w}$ the percentage points were derived by using the Monte Carlo method via the simulation. A power comparison was performed between the FCRE and WCRE and other tests, where, by changing the value of $q$, we indicated when the test has higher and lower power compared with the other tests.

\section{Data Availability}

The simulated data used to support the findings of this study are included within the article.

\section{Conflicts of Interest}

The authors declare that they have no conflicts of interest concerning the publication of this article.

\section{References}

[1] M. Rao, Y. Chen, B. C. Vemuri, and F. Wang, "Cumulative residual entropy: a new measure of information," IEEE Transactions on Information Theory, vol. 50, no. 6, pp. 1220-1228, 2004.

[2] M. A. Abd Elgawad, M. A. Alawady, H. M. Barakat, and S. Xiong, "Concomitants of generalized order statistics from huang-kotz farlie-gumble-morgenstern bivariate distribution: some information measures," Bulletin of the Malaysian Mathematical Sciences Society, vol. 43, no. 3, pp. 2627-2645, 2020.

[3] M. A. Abd Elgawad, H. M. Barakat, S. Xiong, and S. A. Alyami, "Information measures for generalized order statistics and their concomitants under general framework from HuangKotz FGM bivariate distribution," Entropy, vol. 23, no. 335, 2021.

[4] A. Ergun, "The multiplicity of eigenvalues of A vectorial singular diffusion equation with discontinuous conditions," East Asian Science, Technology and Society, vol. 6, no. 2, pp. 22-34, 2019.

[5] A. Ergün, "A half-inverse problem for the singular diffusion operator with jump conditions," Miskolc Mathematical Notes, vol. 21, no. 2, pp. 805-821, 2020. 
[6] Z. A. Abo-Eleneen, "The entropy of progressively censored samples," Entropy, vol. 13, no. 2, pp. 437-449, 2011.

[7] M. A. Alawady, H. M. Barakat, and M. A. Abd Elgawad, "Concomitants of generalized order statistics from bivariate cambanis family of distributions under a general setting," The Bulletin of the Malaysian Mathematical Society Series 2, 2021.

[8] H. M. Barakat and I. A. Husseiny, "Some information measures in concomitants of generalized order statistics under iterated Farlie-Gumbel-Morgenstern bivariate type," Quaestiones Mathematicae, vol. 44, 2020.

[9] E. J. Dudewicz and E. C. Van der Meulen, "Entropy-based tests of uniformity," Journal of the American Statistical Association, vol. 76, no. 376, pp. 967-974, 1981.

[10] M. Mohamed, "A measure of inaccuracy in concomitants of ordered random variables under Farlie-Gumbel-Morgenstern family,” Filomat, vol. 33, no. 15, pp. 4931-4942, 2019.

[11] M. S. Mohamed, "On cumulative residual Tsallis entropy and its dynamic version of concomitants of generalized order statistics," Communications in Statistics-Theory and Methods, 2020.

[12] M. Mirali, S. Baratpour, and V. Fakoor, "On weighted cumulative residual entropy," Communications in StatisticsTheory and Methods, vol. 46, no. 6, pp. 2857-2869, 2017.

[13] M. Mirali and S. Baratpour, "Dynamic version of weighted cumulative residual entropy," Communications in StatisticsTheory and Methods, vol. 46, no. 22, pp. 11047-11059, 2017.

[14] H. A. Noughabi, "Cumulative residual entropy applied to testing uniformity," Communications in Statistics-Theory and Methods, 2020.

[15] R. Amirov, A. Ergun, and S. Durak, "Half-inverse problems for the quadratic pencil of the Sturm-Liouville equations with impulse," Numerical Methods for Partial Differential Equations, vol. 37, no. 1, pp. 915-924, 2021.

[16] H. Xiong, P. Shang, and Y. Zhang, "Fractional cumulative residual entropy," Communications in Nonlinear Science and Numerical Simulation, vol. 78, Article ID 104879, 2019.

[17] J. Yu, W. Gui, and Y. Shan, "Statistical inference on the Shannon entropy of inverse weibull distribution under the progressive first-failure censoring," Entropy, vol. 21, no. 12, p. 1209, 2019.

[18] M. A. Stephens, "EDF statistics for goodness of fit and some comparisons," Journal of the American Statistical Association, vol. 69, no. 347, pp. 730-737, 1974.

[19] H. G. Tucker, "A generalization of the glivenko-cantelli theorem," The Annals of Mathematical Statistics, vol. 30, no. 3, pp. 828-830, 1959.

[20] B. C. Arnold, N. Balakrishnan, and H. N. Nagaraja, A First Course in Order Statistics, Wiley, Hoboken, NJ, USA, 1992.

[21] P. Billingsley, Probability and Measure, John Wiley \& Sons, Hoboken, NJ, USA, 2008.

[22] B. Jóhannesson and N. Giri, "On approximations involving the beta distribution," Communications in Statistics-Simulation and Computation, vol. 24, no. 2, pp. 489-503, 1995. 